\title{
Preoperative statin therapy and infectious complications in cardiac surgery
}

\author{
N. L. Hartholt • T. C. D. Rettig • M. Schijffelen • \\ W. J. Morshuis • E. M. W. van de Garde • P. G. Noordzij
}

Published online: 24 July 2014

(C) The Author(s) 2014. This article is published with open access at Springerlink.com

\begin{abstract}
Aim To assess whether preoperative statin therapy is associated with the risk of postoperative infection in patients undergoing cardiac surgery.

Methods 520 patients undergoing cardiac surgery in 2010 were retrospectively examined. Data regarding statin and antibiotic use prior to and after surgery were available from the hospital pharmacy information system. Cultures and clinical data of patients on postoperative antibiotics other than standard prophylactic therapy were studied to identify postoperative infections up to 30 days from day of surgery.

Results 370 (71.2\%) patients were on preoperative statin therapy. Overall, 82 patients (15.8\%) suffered from postoperative infection of which 11 were surgical site infections. In multivariable regression analysis, statin therapy was associated with a reduced risk of postoperative infection (adjusted odds ratio: $0.329,95 \%$ : CI $0.19-0.57 ; P<0.001$ ).

Conclusions Preoperative statin use was associated with a considerable reduced risk of postoperative infections following
\end{abstract}

N. L. Hartholt and T. C. D. Rettig contributed equally to this study

N. L. Hartholt • E. M. W. van de Garde

Department of Clinical Pharmacy, St. Antonius Hospital,

Koekoekslaan 1, 3430, EM Nieuwegein, the Netherlands

T. C. D. Rettig • P. G. Noordzij ( $\square)$

Department of Anesthesiology, Intensive Care and Pain Medicine,

St. Antonius Hospital, Koekoekslaan 1, 3430, EM Nieuwegein,

the Netherlands

e-mail: p.noordzij@antoniusziekenhuis.nl

M. Schijffelen

Department of Medical Microbiology and Immunology, St. Antonius

Hospital, Koekoekslaan 1, 3430, EM Nieuwegein, the Netherlands

W. J. Morshuis

Department of Cardiothoracic Surgery, St. Antonius Hospital,

Koekoekslaan 1, 3430, EM Nieuwegein, the Netherlands cardiac surgery. Randomised controlled trials are required to clarify the role of statin therapy in the prevention of postoperative infections.

Keywords Statins $\cdot$ Postoperative infection $\cdot$ Cardiac surgery $\cdot$ Surgical site infection

\section{Introduction}

Annually, approximately 16,000 patients are scheduled for cardiothoracic surgery in the Netherlands. [1] Postoperative infections are important causes of increased length of hospital stay and mortality. Despite prophylactic antibiotic therapy, the incidence of postoperative infectious complications is 5 to $21 \%[2-4]$.

In a search for additional ways to decrease the postoperative infection rate, statin therapy is gaining increasing attention. In addition to cholesterol-lowering characteristics, statins have anti-inflammatory, immunomodulatory, antioxidant and antiapoptotic effects that could alter the response to infections. [5] Whether statins have a beneficial effect on postoperative infectious complications in patients undergoing cardiac surgery is unknown, as prior studies have shown conflicting results [6-8].

This study aims to assess whether preoperative statin therapy is associated with the risk of postoperative infection in patients undergoing cardiac surgery.

\section{Methods}

Study population

The total population of 1868 adult patients who had undergone cardiac surgery in the St. Antonius Hospital, Nieuwegein, the 
Netherlands in 2010 was the target population for this study. From this population we selected all patients with data on preoperative drug use in the hospital pharmacy information system. This means that only patients who were hospitalised more than $24 \mathrm{~h}$ before the day of surgery could be studied $(n=593)$, as these are the patients with reviewed preoperative medication profiles available in the hospital pharmacy information system. Additionally, 73 patients were excluded as they were on antimicrobial therapy prior to their surgical procedure outside the routine antimicrobial prophylaxis (cefazolin $2 \mathrm{~g}$ intravenously before skin incision followed by additional cefazolin every four hours for the duration of the procedure and continued up to $48 \mathrm{~h}$ for patients with valve or aortic surgery with exogenous materials). Finally, all patients with a statin in the preoperative medication profile were considered exposed at the time of surgery and all other patients were classified as controls. The local Medical Ethics Committee (Research and Development Department, St. Antonius Hospital) approved the study.

\section{Outcome assessment}

The primary outcome was postoperative infection within 30 days of surgery. We used the following approach to determine if a postoperative infection was present: first, all patients on postoperative antibiotic therapy, aside from perioperative antibiotic prophylaxis, were identified. Secondly, N.H. and T.R. independently examined the medical records of these patients to determine if clinical data (e.g. fever, white cell count) supported the diagnosis of infection. When N.H. and T.R. agreed that a postoperative infection was present, it was classified as a surgical site or non-surgical site infection. Subsequently, M.S. and T.R. used microbiological data to identify the microbial aetiology. When N.H., T.R. and M.S. could not reach an agreement on whether an infection was present or on the site of infection, P.N. was consulted. For all patients, in-hospital mortality was noted.

\section{Potential confounders}

To be able to control for potential confounding, medical information was obtained on risk factors for infection that could potentially confound the association between statin treatment and outcome. For each patient we evaluated the presence of the following comorbidities as potential confounders: diabetes, chronic obstructive pulmonary disease (COPD, graded according to the GOLD classification [9] where all smokers were considered class I unless otherwise stated), peripheral artery disease, three-vessel coronary artery disease, renal insufficiency (defined as serum creatinine $>120 \mu \mathrm{mol} / \mathrm{l}$ ), heart failure (according to New York Heart Association functional classification), prior myocardial infarction, and history of cardiac surgery. In addition, the following surgery-related parameters were assessed: type of surgery (coronary artery bypass graft $(\mathrm{CABG})$, valve surgery, aortic surgery or other), urgent procedure (patients not electively admitted for operation who require surgery before hospital discharge), perfusion time, aortic clamp time and whether rethoracotomy has taken place. Aspirin, antidiabetic agents, beta-blockers, angiotensin-converting enzyme (ACE) inhibitors, proton pump inhibitors (PPIs) and prednisone were examined as possible confounding drugs. A patient was considered exposed to a drug if it was listed in the preoperative medication profile. Furthermore, we calculated the body mass index and European System for Cardiac Operative Risk Evaluation (EURO) score as proxy for overall health status and complication risk. During the study period, glucose values were standardly targeted at $4.5-8 \mathrm{mmol} / 1$ during surgery and ICU admission, and $4.5-10 \mathrm{mmol} / \mathrm{l}$ on the surgical ward in all patients.

\section{Statistical analysis}

Data were analysed using International Business Machines Statistical Package for the Social Science (IBM SPSS) Statistics 19.0 software. Univariate analysis by $\chi^{2}$ tests and Student's $t$ tests was used to test for significant differences in characteristics between statin users and non-users and patients with and without postoperative infections. Multivariable logistic regression analysis was used to estimate the strength of the association between statin treatment and the risk of postoperative infection and expressed as odds ratios (OR) with $95 \%$ confidence intervals (CI). First, we included all potential confounders and age and gender in a multivariable model if a variable was imbalanced $(P<0.10)$ related to either statin use or occurrence of infection. Second, a final model was obtained after stepwise backward elimination of potential confounders if they did not alter the OR by more than $10 \%$. Stratified analyses were conducted to detect possible differences in effects related to timing of statin exposure (continued use, commenced use or discontinued use). For all tests, a $P$-value of 0.05 was considered significant.

\section{Results}

A total of 370 (71.2\%) patients received statin treatment until the day of surgery, while 150 (28.8\%) patients did not. Patients receiving statin treatment were more likely to be male, and suffering from COPD, diabetes, peripheral artery disease, and three-vessel coronary artery disease. Furthermore, they experienced more myocardial infarction and isolated $\mathrm{CABG}$ surgery and received more frequent other cardiovascular drug therapies, such as aspirin, beta-blockers and 
ACE-inhibitors. Compared with non-users, patients on statin therapy had shorter perfusion times and aortic clamp times. Baseline characteristics are shown in Table 1.

Overall, $82(15.8 \%)$ patients suffered from postoperative infection. Of the patients with an infectious complication, $87 \%$ had a non-surgical site infection. The occurrence of a surgical site infection was less common and was present in $13 \%$ of patients with postoperative infection. Pneumonia was the most common infection and accounted for $59 \%$ of all infectious complications. In $59(72 \%)$ patients with a postoperative infection, one or more microorganisms were detected. Characteristics of patients with and without postoperative infection are shown in Table 2.

Patients on preoperative statin therapy suffered less frequently from postoperative infection compared with nonusers $(12.2 \%$ versus $24.7 \%$, respectively; adjusted odds ratio (AOR): 0.329, $95 \%$ CI: 0.19-0.57; $P<0.001$; Table 3). Statin users had fewer non-surgical site infections compared with non-users (AOR: 0.253, 95 \% CI: 0.14-0.45; $P<0.001$ ). This was mainly caused by a reduction in postoperative pneumonia
Table 1 Patient characteristics according to the use of statins
Dichotomous variables are shown as number (\%) and continuous variables are presented as mean $\pm \mathrm{SD}$

\begin{tabular}{|c|c|c|c|}
\hline Variable & $\begin{array}{l}\text { Statin group } \\
(n=370)\end{array}$ & $\begin{array}{l}\text { Control group } \\
(n=150)\end{array}$ & $p$-value \\
\hline \multicolumn{4}{|l|}{ Patient characteristics } \\
\hline Sex (male) & $260(70.3)$ & $87(58.0)$ & 0.007 \\
\hline Age (years) & $68.3 \pm 10.0$ & $69.5 \pm 13.7$ & 0.277 \\
\hline BMI $\left(\mathrm{kg} / \mathrm{m}^{2}\right)$ & $27.7 \pm 4.5$ & $26.7 \pm 4.9$ & 0.037 \\
\hline COPD & $206(55.7)$ & $70(46.7)$ & 0.062 \\
\hline Diabetes & $112(30.3)$ & $27(18.0)$ & 0.004 \\
\hline Congestive heart failure & $140(37.8)$ & $75(50.0)$ & 0.011 \\
\hline Renal failure & $56(15.1)$ & $28(18.7)$ & 0.322 \\
\hline Peripheral artery disease & $79(21.4)$ & $19(12.7)$ & 0.018 \\
\hline Three-vessel coronary artery disease & $209(56.5)$ & $30(20.0)$ & $<0.001$ \\
\hline EURO score & $5.2 \pm 3.7$ & $5.7 \pm 3.3$ & 0.106 \\
\hline Previous myocardial infarction & $180(48.6)$ & $24(16.0)$ & $<0.001$ \\
\hline Previous cardiac surgery & $39(10.5)$ & $23(15.3)$ & 0.127 \\
\hline \multicolumn{4}{|l|}{ Preoperative medication } \\
\hline Beta-blocker & $293(79.2)$ & $91(60.7)$ & $<0.001$ \\
\hline ACE-inhibitor & $163(44.1)$ & $47(31.3)$ & 0.007 \\
\hline Prednisone & $43(11.6)$ & $16(10.7)$ & 0.756 \\
\hline Proton pump inhibitor & $161(43.5)$ & $60(40.0)$ & 0.463 \\
\hline Aspirin & $150(40.5)$ & $23(15.3)$ & $<0.001$ \\
\hline \multicolumn{4}{|l|}{ Postoperative medication } \\
\hline Statin & $349(94.3)$ & $37(24.7)$ & $<0.001$ \\
\hline Beta-blocker & $301(81.4)$ & $110(73.3)$ & 0.056 \\
\hline ACE-inhibitor & $168(45.4)$ & $68(45.3)$ & 0.968 \\
\hline Prednisone & $35(9.5)$ & $21(14.0)$ & 0.126 \\
\hline Proton pump inhibitor & $272(73.5)$ & $103(68.7)$ & 0.291 \\
\hline \multicolumn{4}{|l|}{ Type of surgery } \\
\hline CABG & $219(59.2)$ & $25(16.7)$ & $<0.001$ \\
\hline Major procedure (other or in addition to $\mathrm{CABG}$ ) & $152(41.1)$ & $124(82.7)$ & $<0.001$ \\
\hline Valve surgery & $110(29.7)$ & $90(60.0)$ & $<0.001$ \\
\hline Aortic surgery & $22(5.9)$ & $28(18.7)$ & $<0.001$ \\
\hline Other cardiac surgery & $9(2.4)$ & $19(12.7)$ & $<0.001$ \\
\hline \multicolumn{4}{|l|}{ Perioperative characteristics } \\
\hline Perfusion time (min) & $88.6 \pm 55.6$ & $126.8 \pm 415.5$ & 0.084 \\
\hline Aortic clamp time (min) & $60.0 \pm 37.9$ & $59.2 \pm 55.4$ & 0.836 \\
\hline Off pump & $12(3.2)$ & $3(2.0)$ & 0.443 \\
\hline Urgent surgery & $33(8.9)$ & $9(6.0)$ & 0.268 \\
\hline Rethoracotomy & $29(7.8)$ & $20(13.3)$ & 0.053 \\
\hline
\end{tabular}


Table 2 Patient characteristics according to the occurrence of postoperative infection
Dichotomous variables are shown as number (\%) and continuous variables are presented as mean $\pm \mathrm{SD}$

\begin{tabular}{|c|c|c|c|}
\hline Variable & $\begin{array}{l}\text { Infection group } \\
(n=82)\end{array}$ & $\begin{array}{l}\text { Control group } \\
(n=438)\end{array}$ & $p$-value \\
\hline \multicolumn{4}{|l|}{ Patient characteristics } \\
\hline Sex (male) & $56(68.3)$ & $291(66.4)$ & 0.744 \\
\hline Age (years) & $70.2 \pm 12.0$ & $68.4 \pm 11.0$ & 0.189 \\
\hline BMI $\left(\mathrm{kg} / \mathrm{m}^{2}\right)$ & $27.8 \pm 4.9$ & $27.3 \pm 4.6$ & 0.393 \\
\hline COPD & $57(69.5)$ & $219(50.0)$ & 0.001 \\
\hline Diabetes & $21(25.6)$ & $118(26.9)$ & 0.803 \\
\hline Congestive heart failure & $41(50.0)$ & $174(39.7)$ & 0.083 \\
\hline Renal failure & $20(24.4)$ & $64(14.6)$ & 0.027 \\
\hline Peripheral artery disease & $18(22.0)$ & $80(18.3)$ & 0.389 \\
\hline Three-vessel coronary artery disease & $40(48.8)$ & $199(45.4)$ & 0.494 \\
\hline EURO score & $6.0 \pm 3.5$ & $5.2 \pm 3.6$ & 0.074 \\
\hline Previous myocardial infarction & $27(32.9)$ & $177(40.4)$ & 0.197 \\
\hline Previous cardiac surgery & $9(11.0)$ & $53(12.1)$ & 0.773 \\
\hline \multicolumn{4}{|l|}{ Preoperative medication } \\
\hline Statin & $45(54.9)$ & $325(74.2)$ & 0.000 \\
\hline Beta-blocker & $58(70.7)$ & $326(74.4)$ & 0.484 \\
\hline ACE-inhibitor & $31(37.8)$ & $179(40.9)$ & 0.604 \\
\hline Prednisone & $15(18.3)$ & $44(10.0)$ & 0.031 \\
\hline Proton pump inhibitor & $34(41.5)$ & $187(42.7)$ & 0.836 \\
\hline Aspirin & $26(31.7)$ & $147(33.6)$ & 0.744 \\
\hline \multicolumn{4}{|l|}{ Postoperative medication } \\
\hline Statin & $45(54.9)$ & $341(77.9)$ & 0.000 \\
\hline Beta-blocker & $60(73.2)$ & $351(80.1)$ & 0.143 \\
\hline ACE-inhibitor & $36(43.9)$ & $200(45.8)$ & 0.756 \\
\hline Prednisone & $13(15.9)$ & $43(9.8)$ & 0.109 \\
\hline Proton pump inhibitor & $53(64.6)$ & $322(73.9)$ & 0.087 \\
\hline \multicolumn{4}{|l|}{ Type of surgery } \\
\hline CABG & $34(41.5)$ & $210(47.9)$ & 0.280 \\
\hline Major procedure (other or in addition to $\mathrm{CABG}$ ) & $47(57.3)$ & $229(52.3)$ & 0.402 \\
\hline Valve surgery & $32(39.0)$ & $168(38.4)$ & 0.909 \\
\hline Aortic surgery & $9(11.0)$ & $41(9.4)$ & 0.649 \\
\hline Other cardiac surgery & $4(4.9)$ & $24(5.5)$ & 0.825 \\
\hline \multicolumn{4}{|l|}{ Perioperative characteristics } \\
\hline Perfusion time (min) & $106.1 \pm 72.8$ & $98.4 \pm 246.9$ & 0.781 \\
\hline Aortic clamp time (min) & $65.6 \pm 50.8$ & $58.7 \pm 42.1$ & 0.185 \\
\hline Off pump & $0(0.0)$ & $15(3.4)$ & 0.089 \\
\hline Urgent surgery & $8(9.8)$ & $34(7.8)$ & 0.543 \\
\hline Rethoracotomy & $11(13.4)$ & $38(8.7)$ & 0.180 \\
\hline
\end{tabular}

and urinary tract infection (Table 4). A surgical site infection occurred in 11 (3\%) patients on statin therapy, whereas nonusers did not develop any. Overall, in-hospital mortality was $4.4 \% ; 3.2 \%$ in patients on preoperative statin therapy and $7.3 \%$ in non-users $(P=0.041)$.

After surgery, statin therapy was initiated in 37 (7.1\%) patients who did not use statins prior to surgery. Postoperative initiation of statin therapy was not associated with a reduced risk of infection (AOR: 0.676, $95 \% \mathrm{CI}$ : 0.26-1.75; $P=0.422$ ).

\section{Discussion}

In this cohort of 520 patients undergoing cardiac surgery, preoperative statin therapy was associated with a $67 \%$ reduced risk of a postoperative infectious complication. The reduction in postoperative infection by preoperative statin use was mainly caused by a reduction in nonsurgical site infections, in particular less pneumonia and urinary tract infections. 
Table 3 Results of multivariate analysis for the effect of statin therapy on postoperative infection rate

\begin{tabular}{llr}
\hline & OR $(95 \% \mathrm{CI})$ & $p$-value \\
\hline Unadjusted & $0.423(0.260-0.687)$ & 0.001 \\
Adjusted for sex & $0.412(0.253-0.673)$ & $<0.001$ \\
$\begin{array}{l}\text { Adjusted for sex and age } \\
\text { Adjusted for sex, age and three-vessel } \\
\quad \text { coronary artery disease }\end{array}$ & $0.417(0.255-0.681)$ & $<0.001$ \\
$\begin{array}{c}\text { Adjusted for sex, age, three-vessel } \\
\quad \text { coronary artery disease and COPD }\end{array}$ & $0.318(0.204-0.596)$ & $<0.001$ \\
$\begin{array}{c}\text { Adjusted for sex, age, three-vessel } \\
\quad \text { coronary artery disease, COPD and } \\
\text { aortic clamp time more than 90 min }\end{array}$ & $0.329(0.189-0.572)$ & $<0.001$ \\
\hline
\end{tabular}

Recently two meta-analyses studied the relationship between statins and outcome in patients undergoing high-risk cardiac procedures (e.g. CABG, valve replacement, percutaneous coronary intervention). Guay et al. included 29 randomised controlled trials in which statin therapy was compared with placebo. [10] Statin therapy reduced the risk of myocardial infarction (risk ratio (RR): $0.48 ; 95 \%$ CI $0.38-0.61 ; P<0.001)$ and there was a trend towards lower mortality (RR: $0.26 ; 95 \%$ CI 0.06-1.02; $P=0.053$ ). Another meta-analysis showed that preoperative statin therapy was associated with improved outcome after $\mathrm{CABG}$, but not after aortic valve replacement. [11] However, postoperative infections were not studied in either studies. Papers investigating the effect of statins on postoperative infection in cardiac surgery patients have shown variable results. In a retrospective cohort study of 1934 patients undergoing CABG and/or valve surgery, $151(7.8 \%)$ patients developed an infection. [6]

Table 4 Incidence of study endpoints in statin and control group

\begin{tabular}{lccc}
\hline & $\begin{array}{c}\text { Statin group } \\
\text { No. (\%) }\end{array}$ & $\begin{array}{l}\text { Control group } \\
\text { No. (\%) }\end{array}$ & $p$-value \\
\hline All & $370(100)$ & $150(100)$ & \\
Postoperative infection & $45(12.2)$ & $37(24.7)$ & 0.001 \\
Surgical site infection & $11(3.0)$ & $0(0.0)$ & n.a.* \\
Mediastinitis & $6(1.6)$ & $0(0.0)$ & \\
Wound infection & $5(1.4)$ & $0(0.0)$ & \\
Non-surgical site infection & $34(9.2)$ & $37(24.7)$ & $<0.001$ \\
Pneumonia & $28(7.6)$ & $20(13.3)$ & \\
Urinary tract infection & $2(0.5)$ & $11(7.3)$ & \\
Sepsis & $3(0.8)$ & $5(3.3)$ & \\
Other & $1(0.3)^{\S}$ & $1(0.3)^{\text {ॠ }}$ & \\
In-hospital mortality & $12(3.2)$ & $11(7.3)$ & 0.041 \\
\hline
\end{tabular}

*n.a.: not available

$\S$ prostatitis

๑ unknown
Preoperative statin use was associated with a risk reduction of $33 \%(P=0.04)$ for postoperative infection. The odds ratios for a non-surgical site infection such as pneumonia (OR: 0.67; $95 \%$ CI: 0.43-1.04), urinary tract infection (OR: $0.65 ; 95 \%$ CI: $0.31-1.39$ ) or bacteraemia (OR: $0.71 ; 95 \%$ CI: $0.32-1.58$ ) tended to be less in patients on statins but did not reach statistical significance. Another retrospective cohort study from Kayani et al. in 6253 patients who underwent $\mathrm{CABG}$ showed statin use as being associated with a $26 \%$ reduction in the postoperative infection rate (OR: $0.74 ; 95 \%$ CI: 0.60 0.90). [12] In contrast, Mohamed et al. studied 7733 patients undergoing cardiac surgery and in that study statin users had similar infection rates to nonusers: $8.1 \%$ versus $8.4 \%$, respectively (AOR: $1.08 ; 95 \%$ CI: 0.89-1.31). [8] The incidence of any specific infection was similar in both groups, except for deep sternal/organ space infection $(16(0.6 \%)$ statin users versus $54(1.1 \%)$ nonusers; $P=0.04)$. Importantly, postoperative pneumonia was not scored; considering the fact that pneumonia was diagnosed in $62 \%$ of all infections in the study by Coleman et al. and $59 \%$ in our study, excluding pneumonia could have influenced the results in the study by Mohamed et al.

We noticed a significant difference in infection type between statin users and nonusers. Patients on statin therapy had fewer non-surgical site infections, mainly caused by a reduced incidence of postoperative pneumonia and urinary tract infection. This finding is consistent with the results of previous studies. Using the United Kingdom (UK) General Practice Research Database, Schlienger et al. performed a retrospective case-control study in non-surgical patients and showed that statin therapy was associated with fewer cases of pneumonia, in particular, fatal pneumonia (OR: $0.47,95 \% \mathrm{CI}$ : $0.25-0.88$ ). [13] Van de Garde et al. showed that in patients with a history of diabetes only, statin users had a significantly reduced risk of pneumonia (OR: 0.49, 95 \% CI: 0.35-0.69). [14] In contrast, in our study, surgical site infections occurred more often in patients on statin therapy than nonusers. This finding has not been described before. Known risk factors for surgical site infections, e.g. diabetes, obesity, prolonged operation time, did not appear to be more common in patients on statins in our study. Unfortunately, due to the small numbers we were not able to further investigate these effects with multivariable analysis. The same applies for potential effects of statins on mortality. Crude significant reduced in-hospital mortality could be observed in our study.

If statin therapy is associated with less postoperative nonsurgical site infections, is there a reasonable explanation? It is known that statins suppress the expression of various cytokines, chemokines and adhesion molecules and modulate coagulation toward a less prothrombotic state, but is there a direct effect on bacterial growth? [5] In an experimental study, mice pretreated with low-dose simvastatin and challenged with Staphylococcus aureus intratracheally or intravenously 
had lower lung bacterial burden compared with mice that were pretreated with placebo. [15] Bacterial killing was enhanced in simvastatin-pretreated mice, while mortality was decreased. Catron et al. studied mice pretreated with lovastatin and intraperitoneally infected with Samonella enterica. [16] Intracellular proliferation of Salmonella enterica was inhibited 6to 10 -fold by lovastatin. In summary, in animals, statins seem to be bactericidal and decrease bacterial proliferation. However, well-conducted human studies remain lacking.

During hospitalisation, a patient's drug treatment is modified frequently. For example, statin therapy can be initiated after surgery or discontinued for the remainder of the postoperative period. In a recent randomised controlled trial in patients admitted with severe sepsis, patients were randomised to atorvastatin treatment or placebo. [17] Patients in whom statin treatment was continued showed improved survival, while new-onset statin treatment did not result in improved outcome. Our study revealed that statin therapy was initiated in 37 (7 \%) patients following surgery. Newly initiated statin therapy, however, was not associated with a reduced chance of developing an infection. These results may imply that the possible protective effect of statin therapy on postoperative infection is not immediately reached after the start of therapy, but that it may take weeks before a beneficial effect is apparent.

Compared with other reports, postoperative infection and mortality rates appeared to be relatively high in our study population. The most plausible explanation for this could be the fact that we applied a broad definition for postoperative infection and that we studied patients who were hospitalised more than one day before surgery. One could hypothesise that these patients represent unstable cases and that these patient have a prolonged risk of nosocomial infections. Unfortunately, we were not able to test this hypothesis in depth because of lacking information on outcomes and antibiotic exposure for the excluded patients. Nevertheless, the final population in the present study represents the population that could benefit from interventions such as initiating statins in hospital before surgery if proven beneficial.

Our study has several limitations. First, the results of our study are limited because the data were derived in a retrospective manner. Second, compared with similar studies, our study population is relatively small. And third, we did not have specific information on the length of statin therapy prior to surgery, which prevented additional analysis on timing of statin exposure.

In conclusion, this study describes a protective association between preoperative statin therapy and postoperative infectious complications in a high-risk cardiac surgical cohort. Randomised controlled trials are required to further clarify the role of statin therapy in preventing postoperative infections in cardiac surgery.
Funding This work was supported by institutional and departmental sources.

Conflict of interests None declared.

Open Access This article is distributed under the terms of the Creative Commons Attribution License which permits any use, distribution, and reproduction in any medium, provided the original author(s) and the source are credited.

\section{References}

1. http://www.hartstichting.nl/9800/13333/13374/hvz in_nederland_2011.

2. De Santo LS, Bancone C, Santarpino G, et al. Microbiologically documented nosocomial infections after cardiac surgery: an 18month prospective tertiary care centre report. Eur J Cardiothorac Surg. 2008:33:666-72.

3. Michalopoulos A, Geroulanos S, Rosmarakis ES, Falagas ME. Frequency, characteristics, and predictors of microbiologically documented nosocomial infections after cardiac surgery. Eur $\mathrm{J}$ Cardiothorac Surg. 2006;29:456-60.

4. Kollef MH, Sharpless L, Vlasnik J, Pasque C, Murphy D, Fraser VJ. The impact of nosocomial infections on patient outcomes following cardiac surgery. Chest. 1997;112:666-75.

5. Terblanche M, Almog Y, Rosenson RS, Smith TS, Hackam DG. Statins and sepsis: multiple modifications at multiple levels. Lancet Infect Dis. 2007;7:358-68.

6. Coleman CI, Lucek DM, Hammond J, White CM. Preoperative statins and infectious complications following cardiac surgery. Curr Med Res Opin. 2007;23:1783-90.

7. Daneman N, Thiruchelvam D, Redelmeier DA. Statin use and the risk of surgical site infections in elderly patients undergoing elective surgery. Arch Surg. 2009;144:938-45.

8. Mohamed R, McAlister FA, Pretorius V, et al. Preoperative statin use and infection after cardiac surgery: a cohort study. Clin Infect Dis. 2009;48:e66-72.

9. Rabe KF, Hurd S, Anzueto A, et al. Global strategy for the diagnosis, management, and prevention of chronic obstructive pulmonary disease: GOLD executive summary. Am J Respir Crit Care Med. 2007; 176:532-55.

10. Guay J, Ochroch EA. Effects of adding statins before surgery on mortality and major morbidity: a meta-analysis. J Cardiothorac Vasc Anesth. 2014;28:255-66.

11. Kuhn EW, Liakopoulos OJ, Stange S, et al. Meta-analysis of patients taking statins before revascularization and aortic valve surgery. Ann Thorac Surg. 2013;96:1508-16.

12. Kayani WT, Bandeali SJ, Lee VV, et al. Association between statins and infections after coronary artery bypass grafting. Int $\mathrm{J}$ Cardiol. 2013; 168:117-20.

13. Schlienger RG, Fedson DS, Jick SS, Jick H, Meier CR. Statins and the risk of pneumonia: a population-based, nested case-control study. Pharmacotherapy. 2007;27:325-32.

14. van de Garde EM, Hak E, Souverein PC, Hoes AW, van den Bosch JM, Leufkens HG. Statin treatment and reduced risk of pneumonia in patients with diabetes. Thorax. 2006;61: 957-61.

15. McDowell SA, Ma Y, Kusano R, Akinbi HT. Simvastatin is protective during staphylococcus aureus pneumonia. Curr Pharm Biotechnol. 2011;12:1455-62. 
16. Catron DM, Lange Y, Borensztajn J, Sylvester MD, Jones BD, Haldar K. Salmonella enterica serovar typhimurium requires nonsterol precursors of the cholesterol biosynthetic pathway for intracellular proliferation. Infect Immun. 2004;72:1036-42.
17. Kruger P, Bailey M, Bellomo R, et al. A multicenter randomized trial of atorvastatin therapy in intensive care patients with severe sepsis. Am J Respir Crit Care Med. 2013;187:743-50. 